\title{
FCNC in top quark transitions in ATLAS
}

\section{Florencia Daneri ${ }^{* \dagger}$}

Universidad de Buenos Aires, Argentina

E-mail: daneriflorencia@gmail.com

\begin{abstract}
There are many flavour changing neutral current (FCNC) searches ongoing that focus on the top quark sector using $p p$ collisions data taken with the ATLAS detector at the LHC. Several couplings and final states are being investigated based on different analysis strategies. No evidence has been found so far and upper limits on decay and production branching ratios were set for many processes, as well as limits on effective couplings. A brief description and the latest results for each of these analyses are presented in this proceeding.
\end{abstract}

18th International Conference on B-Physics at Frontier Machines - Beauty2019 -

29 September / 4 October, 2019

Ljubljana, Slovenia

\footnotetext{
*Speaker.

${ }^{\dagger}$ On behalf on the ATLAS Collaboration
} 


\section{Introduction}

The top quark is the heaviest elementary particle known, with a mass $m_{t}=173.3 \pm 0.8 \mathrm{GeV}$ [1]. In the Standard Model of Particle Physics (SM), it decays almost exclusively into a $b$ quark and a $W$ boson while flavour-changing neutral current (FCNC) decays are forbidden at tree level. FCNC decays occur at one-loop level but are strongly suppressed by the GIM mechanism [2] with a suppression factor of 14 orders of magnitude relative to the dominant decay mode [3]. However, in several SM scenarios GIM suppresion can be relaxed and/or some particles can contribute to the loops yielding effective couplings that are orders of magnitude larger than those of the SM [4], reaching observable levels. These interactions lead to various top decay and single top production processes. Observation of such processes, extremely rare in the SM, would provide a clear indirect signal of new physics, hence the importance of these analyses. Examples of such beyond the standard model theories are the quark-singlet model (QS) [5], the two-Higgs-doublet model with (FC 2HDM) or without (2HDM) flavour conservation [6], the Minimal Supersymmetric Standard Model (MSSM) [7], the MSSM with R-parity violation (RPV SUSY) [8], models with warped extra dimensions (RS) [9], or extended mirror fermion models (EMF) [10].

Profiting from the abundant $t \bar{t}$ final states produced in $p p$ collisions at LHC, the ATLAS experiment [11] has been carrying out several analyses focusing on the top quark sector in the search for evidence of FCNC. Both, the $t \bar{t}$ and single top production, are contemplated in these searches. A brief description of the analysis strategies and the results for each search is presented next.

\section{Top pair production}

Within the ATLAS experiment, many new physics searches are performed using top-quark events, with one top quark decaying through the $t \rightarrow Z q$ or $t \rightarrow H q$ (q=u,c) FCNC channel, and the other through the dominant SM mode $t \rightarrow b W$. Data collected from $p p$ collisions at a centre-ofmass energy of $\sqrt{s}=13 \mathrm{TeV}$, corresponding to an integrated luminosity of $36.1 \mathrm{fb}^{-1}$, are analysed for these searches and experimental limits on the FCNC branching ratio (BR) are established separately for each coupling. A few more details of each analysis are detailed below.

\section{$2.1 t \rightarrow Z q$}

One of the FCNC searches in top-quark decays analyses the $t \rightarrow Z q$ [12] coupling. Only $Z$ boson decays into charged leptons and leptonic $W$ boson decays are considered as signal. Thus, the final-state topology is characterised by the presence of three isolated charged leptons (electrons or muons), at least two jets, with exactly one being tagged as a jet containing $b$-hadrons, and missing transverse momentum from the undetected neutrino.

A further kinematic selection [12] is applied to keep events with this topology, and $Z$ boson candidates are reconstructed from the two leptons that have the same flavour, opposite charge, and a reconstructed mass within $15 \mathrm{GeV}$ of the $Z$ boson mass.

Applying energy-momentum conservation, the kinematic properties of the top quarks are reconstructed from the corresponding decay particles by minimising 


$$
\chi^{2}=\frac{\left(m_{j_{a} \ell_{a} \ell_{b}}^{\mathrm{reco}}-m_{t_{\mathrm{FCNC}}}\right)^{2}}{\sigma_{t_{\mathrm{FCNC}}}^{2}}+\frac{\left(m_{j_{b} \ell_{c} v}^{\mathrm{reco}}-m_{\mathrm{tSM}_{\mathrm{S}}}\right)^{2}}{\sigma_{t_{\mathrm{SM}}}^{2}}+\frac{\left(m_{\ell_{c} v}^{\mathrm{reco}}-m_{t_{W}}\right)^{2}}{\sigma_{t_{W}}^{2}}
$$

where $m_{j_{a} \ell_{a} \ell_{b}}^{\text {reco }}, m_{j_{b} \ell_{c} v}^{\text {reco }}$ and $m_{\ell_{c} v}^{\text {reco }}$ are the reconstructed masses of the $q Z, b W$ and $\ell v$ systems, respectively. For each jet combination, $j_{b}$ corresponds to the $b$-tagged jet, while any jet can be assigned to $j_{a}$. The longitudinal component of the neutrino momentum $\left(p_{v}^{z}\right)$ is then determined by the minimisation of $\chi^{2}$. The central values of the masses and the widths of the top quarks and the $W$ boson are taken from simulated signal events. The combination with the minimum $\chi^{2}$ is chosen, which fixes the assignment of reconstructed particles and the corresponding $p_{v}^{z}$ value. The final requirements defining the signal region $(\mathrm{SR})$ are $\left|m_{j_{a} \ell_{a} \ell_{b}}^{\text {reco }}-172.5 \mathrm{GeVl}<40 \mathrm{GeV},\right| m_{j_{b} \ell_{c} v}^{\text {reco }}-172.5$ $\mathrm{GeVl} \mid<40 \mathrm{GeV}$, and $\mid m_{\ell_{c} v}^{\text {reco }}-80.4 \mathrm{GeVl}<30 \mathrm{GeV}$.

The main sources of background events containing three prompt leptons are diboson, $t \bar{t} Z$, and $t Z$ production. Events with two or fewer prompt leptons and additional non-prompt ${ }^{1}$ leptons are also sources of background. Besides the signal region, control regions (CR) are defined using various techniques to constrain the main backgrounds.

The main uncertainties, in both the background and signal estimations, are from theoretical normalisation uncertainties and uncertainties in the modelling of background processes in the simulation.

Results are obtained using a binned likelihood fit to kinematic distributions in the signal and control regions. The inclusion of the CRs in a combined fit with the SR constrains the backgrounds and reduces systematic uncertainties. The kinematic distributions used in the fit are the $\chi^{2}$ of the kinematical reconstruction for the SR, the leading lepton's $p_{\mathrm{T}}$ for the non-prompt leptons and $t \bar{t} Z$ CRs, the transverse mass for the $W Z \mathrm{CR}$, and the reconstructed mass of the four leptons for the $Z Z$ CR.

A stastical analysis is performed and since no evidence of a signal is found, 95\% CL limits on the observed(expected) $t \rightarrow Z q$ BR are established: $\mathscr{B}(t \rightarrow Z u)<1.7(2.4) \times 10^{-4}$ and $\mathscr{B}(t \rightarrow$ $Z c)<2.4(3.2) \times 10^{-4}$.

\section{$2.2 t \rightarrow H q$}

The other prominent FCNC search focuses on top quarks decaying into $u$ or $c$ quarks with the emission of a Higgs boson. Depending on the subsequent Higgs decay, four signatures are accesible to the experiment and each one presents a dedicated analysis. Finally, a combined interpretation of these results is performed by ATLAS.

\subsection{1 $t \rightarrow H\left(W W^{*} / Z Z^{*}\right) q$}

One of these signatures targets multilepton final states [13]. Two categories are defined: two same-charge light leptons ( $e$ or $\mu$ ) with four or more jets, of which one or two must be $b$-tagged

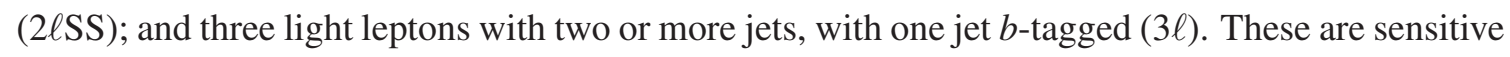
primarily to $H \rightarrow W W^{*}$ decays, with subleading contributions from $H \rightarrow \tau \tau$ and $H \rightarrow Z Z^{*}$.

\footnotetext{
${ }^{1}$ Prompt leptons are leptons from the decay of $W$ or $Z$ bosons, either directly or through an intermediate $\tau \rightarrow \ell v v$ decay, or from the semileptonic decay of top quarks.
} 
The largest sources of background are those arising from non-prompt leptons (from hadron decays, photon conversions, and charge miss-assignment), mainly from $t \bar{t}$ decays, and prompt lepton backgrounds from $t \bar{t} V$ production $(V=W$ or $Z$ ) with leptonic decays of the vector boson. These are estimated using simulation and data-driven techniques.

The FCNC processes are simulated and specialised boosted decision trees (BDT) using the kinematic properties of the final-state particles are used to distinguish FCNC signals from nonprompt lepton backgrounds and from $t \bar{t} W$ and $t \bar{t} Z$ production. BDTs are trained separately in the two categories and for each case, two separate discriminants are trained: one to separate $t \rightarrow H q$ from non-prompt leptons and one to separate $t \rightarrow H q$ from $t \bar{t} V$ processes. Finally, all the results are combined.

Inputs to the BDTs include lepton flavour and kinematic observables, jet properties including whether they are $b$-tagged, angular separations between objects, the $E_{\mathrm{T}}^{\text {miss }}$, and the quantity $m_{\text {eff }}=$ $E_{\mathrm{T}}^{\text {miss }}+H_{\mathrm{T}}$, where $H_{\mathrm{T}}$ is the scalar sum of the $p_{\mathrm{T}}$ of the leptons and jets in the event. Signal events can be distinguished from SM background by having only one true $b$-jet and being relatively soft events with low $m_{e f f}$ and $H_{\mathrm{T}}$.

Binned maximum-likelihood fits to the distributions of the $2 \ell S S$ and $3 \ell$ FCNC discriminants are performed to extract the best-fit values of the $t \rightarrow H q$ branching fractions. Since no evidence of FCNC decays is found, upper limits are set on the observed(expected) BR: $\mathscr{B}(t \rightarrow H u)<$ $1.6(1.5) \times 10^{-3}$ and $\mathscr{B}(t \rightarrow H c)<1.9(1.5) \times 10^{-3}$ at $95 \% \mathrm{CL}$.

\subsection{2 $t \rightarrow H(b \bar{b}) q$}

Another analysis focuses on the $H \rightarrow b \bar{b}$ decay [15], exploiting the high multiplicity of $b$ quarks in the final state.

Only leptonically decaying $W \mathrm{~s}$ are considered, so signal is expected to have one isolated lepton ( $e$ or $\mu$ ) and at least four jets in the final state, three of them originating from $b$-quarks, which can be effectively exploited to suppress the background which mainly comes from $t \bar{t}$ and heavy flavour jets.

In order to optimise the sensitivity of the search, the selected events are categorised into different analysis regions depending on the number of jets $(4,5$ and $\geq 6)$ and on the number of $b$-tagged jets $(2,3$ and $\geq 4)$. Therefore, a total of nine analysis regions is considered. In this way, regions with exactly two $b$-tagged jets are dominated by $t \bar{t}+$ light-jets, while regions with at least four are dominated by $t \bar{t}+\geq 1 b$. Intermediate compositions are found in regions with exactly three $b$-tagged jets. Regions with four or five jets and exactly three $b$-tagged jets dominate the sensitivity of this search.

The main systematic uncertainties arise from the background modelling and the $c$-jet mistagging.

This search uses a likelihood (LH) discriminant to separate signal and background events. The LH variable for a given event is defined as:

$$
L(\mathbf{x})=\frac{P^{\mathrm{sig}}(\mathbf{x})}{P^{\operatorname{sig}}(\mathbf{x})+P^{\mathrm{bkg}}(\mathbf{x})}
$$

where $P^{\mathrm{sig}}(\mathbf{x})$ and $P^{\mathrm{bkg}}(\mathbf{x})$ stand for the probability density functions (pdf) of a given event under the signal hypothesis and under the background hypothesis, respectively. Both are functions 
of $\mathbf{x}$, representing the four-momentum vectors of all final-state particles at the reconstruction level: the lepton, the missing transverse momentum, and the selected jets in a given analysis region. The value of the multivariate $b$-tagging discriminant for each jet is also included in $\mathbf{x}$.

Finally, a binned likelihood fit under the signal-plus-background hypothesis is performed on the LH discriminant distributions in the nine analysis regions considered.

In the absence of a significant excess of data events above the background expectation, 95\% CL observed(expected) limits are set on the BR: $\mathscr{B}(t \rightarrow H u)<5.2(4.9) \times 10^{-3}$ and $\mathscr{B}(t \rightarrow H c)<$ $4.2(4.0) \times 10^{-3}$.

\subsection{3 $t \rightarrow H\left(\tau^{+} \tau^{-}\right) q$}

The other Higgs boson fermionic decay considered is $H \rightarrow \tau^{+} \tau^{-}$[15]. The signal is characterised by the presence of $\tau$-leptons from the decay of the Higgs boson and at least four jets, only one of which originates from a $b$-quark. If one of the $\tau$-leptons decays leptonically, an isolated electron or muon and significant $E_{\mathrm{T}}^{\text {miss }}$ are also expected. However, in a significant fraction of the events the lowest- $p_{\mathrm{T}}$ jet from the $W$ boson decay fails the minimum $p_{\mathrm{T}}$ requirement, resulting in signal events with only three jets reconstructed. In order to optimise the sensitivity of the search, the selected events are categorised into four SRs depending on the number of $\tau$-lep and $\tau$-had candidates, and on the number of jets: ( $\tau$-lep $\tau$-had, $3 \mathrm{j}),(\tau$-lep $\tau$-had, $\geq 4 \mathrm{j}),(\tau$-had $\tau$-had, $3 \mathrm{j})$, and ( $\tau$-had $\tau$-had, $\geq 4 \mathrm{j}$ ). This event categorisation is primarily motivated by the different quality of the event kinematic reconstruction, depending on the amount of ${ }_{\mathrm{T}}^{E m i s s}$ in the event, and whether a jet from the hadronic top-quark decay is missing or not.

The main background comes from fake leptons, estimated using data-driven techniques on CRs. Their modelling also represents the main systematic uncertainty for this search.

BDTs are used in each SR to improve the separation between signal and background. They used kinematic variables as inputs, mainly reconstructed masses of the objects as well as their $p_{\mathrm{T}}$ and the $E_{\mathrm{T}}^{\text {miss }}$.

Then, a binned likelihood fit under the signal-plus-background hypothesis is performed on the BDT discriminant distributions in the four analysis regions considered.

Since no significant excess of data events above the background expectation is found, observed(expected) limits at 95\% CL are set on BR: $\mathscr{B}(t \rightarrow H u)<1.7(2.0) \times 10^{-3}$ and $\mathscr{B}(t \rightarrow$ $H c)<1.9(2.1) \times 10^{-3}$. These results are dominated by the $\tau$-had $\tau$-had channel, which has a sensitivity a factor of two better than that of the $\tau$-lep $\tau$-had channel.

\subsection{4 $t \rightarrow H(\gamma \gamma) q$}

The last signature refers to a Higgs decay into two photons [14]. The search is split into two categories depending on the decay nature of the $W$ boson from the SM top quark decay. In both cases, the final state is characterised by the presence of two photons. For the hadronic category, at least four jets are expected and at least one must be $b$-tagged. While for the leptonic one, at least two jets are expected, as well as one lepton and $E_{\mathrm{T}}^{\text {miss }}$.

The largest backgrounds are $t \bar{\gamma} \gamma, W \gamma \gamma$ and $\gamma \gamma+$ jets in the leptonic case, while only the last one affects the hadronic one. All these backgrounds are estimated using data-driven techniques.

The analysis strategy is similar for both categories, in order to verify if the final state particles are kinematically compatible with the decay of two top quarks, three-body objects are formed: 
the two photons plus one jet on one side (Top1), and the three other jets on the other side or the remaining jet, the lepton, and the neutrino (Top2) depending on the $W$ decaying mode. Hence, (Top1,Top2) pairs are constructed with corresponding invariant masses (M1,M2). For an event to be selected, there must be at least one combination (Top1,Top2) with masses (M1,M2) compatible with the top quark mass. By studying these distributions, selection range windows are chosen for M1 and M2 in each category.

For the hadronic category, an unbinned likelihood fit is performed to the diphoton invariant mass spectra (already constrained by the selection mass window). While for the leptonic, the observed number of events is so small after the selection that an event counting is performed instead.

No significant excess is observed and an upper limits are set on the observed(expected) BR: $\mathscr{B}(t \rightarrow H u)<1.7(2.4) \times 10^{-3}$ and $\mathscr{B}(t \rightarrow H c)<2.2(1.6) \times 10^{-3}$ at the $95 \% \mathrm{CL}$. These results are also translated into limits on the off-diagonal Yukawa coupling [14] where the corresponding limit on the $t c H$ coupling is 0.090 at the $95 \%$ CL.

\subsection{5 $t \rightarrow H q$ Combination}

The $t q H(b \bar{b})$ and $t q H(\tau \tau)$ searches are combined with the ATLAS searches in diphoton and multilepton final states of events in the same data set and the results are shown in Figure 1.
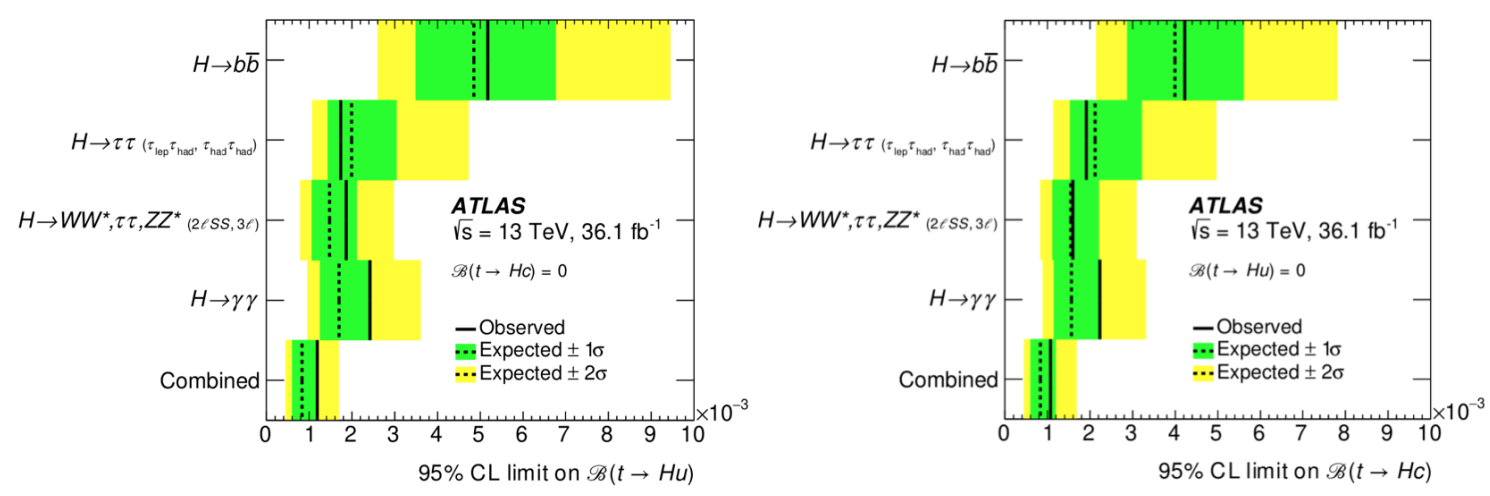

Figure 1: $95 \% \mathrm{CL}$ upper limits on $\mathscr{B}(t \rightarrow H q)$ for the individual searches as well as their combination. The observed limits (solid lines) are compared with the expected (median) limits under the backgroundonly hypothesis (dotted lines). The surrounding shaded bands correspond to the $68 \%$ and $95 \%$ CL intervals around the expected limits, denoted by $\pm 1 \sigma$ and $\pm 2 \sigma$, respectively.

The final combination sets the following observed (expected) limits on the BR: $\mathscr{B}(t \rightarrow H u)<$ $1.2(0.83) \times 10^{-3}$ and $\mathscr{B}(t \rightarrow H c)<1.1(0.83) \times 10^{-3}$.

Upper limits on the branching ratios are also translated into upper limits on the non-flavourdiagonal Yukawa couplings [15] $\lambda_{t q H}$, resulting in $\left|\lambda_{t c H}\right|<0.064(0.055)$ and $\left|\lambda_{t u H}\right|<0.066(0.055)$.

\section{Single top production}

The ATLAS Collaboration also performs searches for single top-quark production via FCNC processes, from gluon or photon plus $u$ or $c$ quark initial states. These two analyses are briefly described in the following sections. Data collected from $p p$ collisions at a centre-of-mass energy 
of $\sqrt{s}=13 \mathrm{TeV}$, corresponding to an integrated luminosity of $81 \mathrm{fb}^{-1}$ are analysed for the $\gamma q \rightarrow t$ coupling; while $20.3 \mathrm{fb}^{-1}$ of $\sqrt{s}=8 \mathrm{TeV}$ data are used for the $g q \rightarrow t$ one.

\section{1 $\gamma q \rightarrow t$}

The search [16] strategy selects events with a final state containing one prompt photon and the decay products of a top quark, namely an electron or a muon, a $b$-tagged jet and missing transverse momentum. With this selection, the search is most sensitive to FCNCs in production mode, but the decay mode is included in the analysis. The main background contribution stems from electrons misidentified as photons, primarily in $t \bar{t}$ events. These contributions and those from hadrons misidentified as photons (both labelled "fakes") are modelled by Monte Carlo (MC) simulations and scaled to data-driven estimates. Also processes with a prompt photon represent an important source of background.

Signal and background events are distinguished using a neural network (NN). Separate NNs are trained for FCNC processes with a $t u \gamma$ or a $t c \gamma$ vertex and with left-handed (LH) or right-handed (RH) couplings. For the signal sample, the production mode was chosen, since the kinematic differences between the production mode and the background are more pronounced than for the decay mode and thus lead to better discrimination between signal and background. Ten variables are inputs to the NN: the $p_{\mathrm{T}}$ of the photon, the lepton and the jet, the charge of the lepton, $E_{\mathrm{T}}^{\text {miss }}$, the lepton-photon and lepton-jet invariant masses, the $\Delta \mathrm{R}$ between the lepton and the photon, between the lepton and the jet, and between the jet and the photon.

Finally, simultaneous binned profile-likelihood fits to the NN-output distribution of the SR and $W+\gamma+$ jet $\mathrm{CR}$ as well as the photon- $p_{\mathrm{T}}$ distribution of the $Z+\gamma \mathrm{CR}$ are performed.

No significant FCNC contributions are observed. From the 95\% CL limits on the signal contribution, the corresponding limits on the effective coupling ${ }^{2}$ parameters [19] are calculated, and from these the limits on the production cross section and BR [17][18] are calculated separately for LH and RH couplings. Results can be seen in Table 1.

\section{$3.2 g q \rightarrow t$}

This search [20] only focuses on the production mode. Events are expected to contain exactly one isolated electron or muon, missing transverse momentum and one jet, which is required to be identified as a jet originating from a $b$-quark. Several SM processes have the same final-state topology and are considered as backgrounds to the FCNC analysis. The main backgrounds are $V+$ jets production (especially in association with heavy quarks), where $V$ denotes a $W$ or a $Z$ boson, SM top-quark production, diboson production, and multijet production via QCD processes. The studied process can be differentiated from SM single top-quark production, which is usually accompanied by additional jets. Furthermore, FCNC production has kinematic differences from the background processes, such as lower transverse momenta of the top quark.

As no single variable provides sufficient discrimination between signal and background events, multivariate analysis techniques are used to separate signal candidates from background candidates. A neural-network (NN) classifier is used with several categories of variables. Apart from basic event kinematics such as the $m_{\mathrm{T}}(W)$ or $H_{\mathrm{T}}$ (the scalar sum of the transverse momenta of all objects

\footnotetext{
${ }^{2}$ The corresponding operators are $O_{u B}^{(i j)}$ and $O_{u W}^{(i j)}$, where $i \neq j$ are indices for the quark generation.
} 


\begin{tabular}{ccccc}
\hline Observable & Vertex & Coupling & Obs. & Exp. \\
\hline$\left|C_{u W}^{(13) *}+C_{u B}^{(13) *}\right|$ & $t u \gamma$ & LH & 0.19 & $0.22_{-0.03}^{+0.04}$ \\
$\left|C_{u W}^{(31)}+C_{u B}^{(31)}\right|$ & $t u \gamma$ & RH & 0.27 & $0.27_{-0.04}^{+0.05}$ \\
$\left|C_{u W}^{(23) *}+C_{u B}^{(23) *}\right|$ & $t c \gamma$ & LH & 0.52 & $0.57_{-0.09}^{+0.11}$ \\
$\left|C_{u W}^{(32)}+C_{u B}^{(32)}\right|$ & $t c \gamma$ & RH & 0.48 & $0.59_{-0.09}^{+0.12}$ \\
\hline$\sigma(p p \rightarrow t \gamma)[\mathrm{fb}]$ & $t u \gamma$ & LH & 36 & $52_{-14}^{+21}$ \\
$\sigma(p p \rightarrow t \gamma)[\mathrm{fb}]$ & $t u \gamma$ & $\mathrm{RH}$ & 78 & $75_{-21}^{+31}$ \\
$\sigma(p p \rightarrow t \gamma)[\mathrm{fb}]$ & $t c \gamma$ & LH & 40 & $49_{-14}^{+20}$ \\
$\sigma(p p \rightarrow t \gamma)[\mathrm{fb}]$ & $t c \gamma$ & $\mathrm{RH}$ & 33 & $52_{-14}^{+22}$ \\
\hline $\mathscr{B}(t \rightarrow q \gamma)\left[10^{-5}\right]$ & $t u \gamma$ & $\mathrm{LH}$ & 2.8 & $4.0_{-1.1}^{+1.6}$ \\
$\mathscr{B}(t \rightarrow q \gamma)\left[10^{-5}\right]$ & $t u \gamma$ & $\mathrm{RH}$ & 6.1 & $5.9_{-1.6}^{+2.4}$ \\
$\mathscr{B}(t \rightarrow q \gamma)\left[10^{-5}\right]$ & $t c \gamma$ & $\mathrm{LH}$ & 22 & $27_{-7}^{+11}$ \\
$\mathscr{B}(t \rightarrow q \gamma)\left[10^{-5}\right]$ & $t c \gamma$ & $\mathrm{RH}$ & 18 & $28_{-8}^{+12}$ \\
\hline
\end{tabular}

Table 1: Observed (expected) 95\% CL limits on the effective coupling strengths for different vertices and couplings, the production cross section, and the branching ratio. For the former, the energy scale is assumed to be $\Lambda=1 \mathrm{TeV}$.

in the final state), various object combinations are considered like the basic kinematic properties of reconstructed objects like the $W$ boson and the top quark, as well as angular distances in $\eta$ and $\phi$ between the reconstructed and final-state objects.

Finally, a binned maximum-likelihood fit is performed to the complete NN output distributions in the signal region. And since no evidence of FCNC processes is seen, limits are set at a 95\%CL to the observed (expected) BR: $\mathscr{B}(t \rightarrow g u)<0.4(0.35) \times 10^{-4}$ and $\mathscr{B}(t \rightarrow g c)<2.0(1.8) \times 10^{-4}$. Also, a limit at a $95 \% \mathrm{CL}$ on the production cross-section multiplied by the branching fraction is established: $\sigma_{q g \rightarrow t} \times \mathscr{B}(t \rightarrow W b)<3.4(2.9)$ pb. Upper limits on the coupling constants divided by the scale of new physics $\Lambda[21] \kappa_{\text {ugt }} / \Lambda<5.8 \times 10^{-3} \mathrm{TeV}^{-1}$ and $\kappa_{\text {cgt }} / \Lambda<13 \times 10^{-3} \mathrm{TeV}^{-1}$ are derived from the observed limit too at the same $95 \% \mathrm{CL}$.

\section{Summary of results}

A strong programme searching for FCNC evidence in the top quark sector is currently undergoing within the ATLAS experiment. Several couplings and final states are investigated and upper limits are set mainly on the BR, and these results are also translated into effective couplings with different interpretations for some searches. A summary of the limit on the BR for each search is shown in Table 2. 


\begin{tabular}{cc}
\hline Observable & Observed (Expected) \\
\hline $\mathscr{B}(t \rightarrow Z u)$ & $1.7(2.4) \times 10^{-4}$ \\
$\mathscr{B}(t \rightarrow Z c)$ & $2.4(3.2) \times 10^{-4}$ \\
\hline $\mathscr{B}(t \rightarrow H u)$ & $1.2(0.83) \times 10^{-3}$ \\
$\mathscr{B}(t \rightarrow H c)$ & $1.1(0.83) \times 10^{-3}$ \\
\hline $\mathscr{B}(t \rightarrow \gamma u) \mathrm{LH}$ & $2.8(4.0) \times 10^{-5}$ \\
$\mathscr{B}(t \rightarrow \gamma u) \mathrm{RH}$ & $6.1(5.9) \times 10^{-5}$ \\
$\mathscr{B}(t \rightarrow \gamma c) \mathrm{LH}$ & $22(27) \times 10^{-5}$ \\
$\mathscr{B}(t \rightarrow \gamma c) \mathrm{RH}$ & $18(28) \times 10^{-5}$ \\
\hline $\mathscr{B}(t \rightarrow g u)$ & $0.4(0.35) \times 10^{-4}$ \\
$\mathscr{B}(t \rightarrow g c)$ & $2.0(1.8) \times 10^{-4}$ \\
\hline
\end{tabular}

Table 2: 95\% CL upper limits on BR for the individual searches and the combination for the Higgs coupling.

\section{References}

[1] ATLAS, CDF, CMS and DØ Collaborations, First combination of Tevatron and LHC measurements of the top-quark mass, ATLAS-CONF-2014-008, 2014

[https://cds.cern.ch/record/1669819].

[2] S. Glashow, J. Iliopoulos and L. Maiani, Weak Interactions with Lepton-Hadron Symmetry, Phys. Rev. D 2 (1970) 1285

[3] J. Aguilar-Saavedra, Top flavor-changing neutral interactions: Theoretical expectations and experimental detection, Acta Phys. Polon. B 35 (2004) 2695 [arXiv: hep-ph/ 0409342 ].

[4] J. A. Aguilar-Saavedra, Top flavour-changing neutral interactions: theoretical expectations and experimental detection, Acta Phys.Polon. B35:2695-2710 [arXiv: hep-ph/0 409342 v 4].

[5] J. Aguilar-Saavedra, Effects of mixing with quark singlets, Phys. Rev. D 69 (2004) 099901 [arXiv:hep-ph/0210112].

[6] D. Atwood, L. Reina and A. Soni, Phenomenology of two Higgs doublet models with flavor changing neutral currents, Phys. Rev. D 55 (1997) 3156 [arXiv: hep-ph/ 9609279 ].

[7] J. Cao, G. Eilam, M. Frank, K. Hikasa, G. Liu et al., SUSY-induced FCNC top-quark processes at the large hadron collider, Phys. Rev. D 75 (2007) 075021 [arXiv: hep-ph/0702264].

[8] J. M. Yang, B.-L. Young and X. Zhang, Flavor changing top quark decays in R-parity-violating SUSY, Phys. Rev. D 58 (1998) 055001 [arXiv: hep-ph/9705341].

[9] K. Agashe, G. Perez and A. Soni, Collider Signals of Top Quark Flavor Violation from a Warped Extra Dimension, Phys. Rev. D 75 (2007) 015002 [arXiv: hep-ph/ 0606293 ].

[10] P. Q. Hung, Y.-X. Lin, C. S. Nugroho and T.-C. Yuan, Top Quark Rare Decays via Loop-Induced FCNC Interactions in Extended Mirror Fermion Model, 2017 [arXiv: 1709.01690].

[11] ATLAS Collaboration, The ATLAS Experiment at the CERN Large Hadron Collider, JINST 3 (2008) S08003.

[12] ATLAS Collaboration, Search for flavour-changing neutral current top-quark decays $t \rightarrow q Z$ in proton-proton collisions at $\sqrt{s}=13 \mathrm{TeV}$ with the ATLAS detector, JHEP 07 (2018) 176 [arXiv:1803.09923v2]. 
[13] ATLAS Collaboration, Search for flavor-changing neutral currents in top quark decays $t \rightarrow H c$ and $t \rightarrow H u$ in multilepton final states in proton-proton collisions at $\sqrt{s}=13 \mathrm{TeV}$ with the ATLAS detector, Phys. Rev. D 98, 032002 (2018) [arXiv: 1805.03483v2].

[14] ATLAS Collaboration, Search for top quark decays $t \rightarrow q H$, with $H \rightarrow \gamma \gamma$, in $\sqrt{s}=13 \mathrm{TeV} p p$ collisions using the ATLAS detector, JHEP 10 (2017) 129 [arXiv: $1707.01404 \mathrm{v} 2$ ].

[15] ATLAS Collaboration, Search for top-quark decays $t \rightarrow H q$ with $36 \mathrm{fb}^{-1}$ of pp collision data at $\sqrt{s}=$ $13 \mathrm{TeV}$ with the ATLAS detector, JHEP 05 (2019) 123 [arXiv: $1812.11568 \mathrm{v} 2]$.

[16] ATLAS Collaboration, Search for flavour-changing neutral currents in processes with one top quark and a photon using $81 \mathrm{fb}^{-1}$ of pp collisions at $\sqrt{\mathrm{s}}=13 \mathrm{TeV}$ with the ATLAS experiment, Phys. Lett. B 800 (2019) 135082 [arXiv: $1908.08461 \mathrm{v} 2$ ].

[17] J. A. Aguilar-Saavedra, A minimal set of top anomalous couplings, Nucl. Phys. B 812 (2009) 181 [arXiv:0811.3842].

[18] G. Durieux, F. Maltoni and C. Zhang, Global approach to top-quark flavor-changing interactions, Phys. Rev. D 91 (2015) 074017 [arXiv: 1412 . 7166 ].

[19] B. Grzadkowski, M. Iskrzynski, M. Misiak and J. Rosiek, Dimension-six terms in the Standard Model Lagrangian, JHEP 10 (2010) 085 [arXiv: 1008 . 4884].

[20] ATLAS Collaboration, Search for single top-quark production via flavour-changing neutral currents at 8 TeV with the ATLAS detector, Eur. Phys. J. C (2016) 76:55 [arXiv:1509.00294v3].

[21] R. Coimbra et al., MEtop - a generator for single top production via FCNC interactions, Eur. Phys. J. C 72 (2012) 2222 [arXiv: 1207.7026$].$ 